\title{
Research on Emergency Communication System
}

\author{
Yuanyuan Guo ${ }^{1, a}$, Yuxin Ou², b, Bin $\mathrm{Liu}^{3, \mathrm{c}}$ \\ ${ }^{1}$ Assistant engineer of the communication tem of General staff Department of the generally team of \\ Fujian Armed Police Force, Fuzhou 350003, China \\ ${ }^{2}$ Maintenance station of Armed Police headquarters, Beijing 100089, China \\ ${ }^{3}$ Assistant engineer of the communication tem of General staff Department of the generally team of \\ Fujian Armed Police Force, Fuzhou 350003, China \\ a523204090@qq.com, b605449661@qq.com, c736587681@qq.com
}

Keywords: Emergency communication system; Emergencies; Emergency communication support.

\begin{abstract}
In recent years, major and unexpected public events in our country have taken place from time to time, causing great losses to the national economy and people's lives and property. Because these emergencies have caused huge damage to various equipments, resulting in the unblocked communication and affecting the rescue and command work, there is still a long way to go to meet the requirements of emergency communications support. Therefore, it is imperative to develop the emergency communication system in China.
\end{abstract}

\section{Introduction}

China is a country with frequent disasters, particularly natural disasters, which have caused great losses to the national economy and people's lives and property. Especially after Wenchuan earthquake, in the face of all kinds of natural disasters and emergencies, implementing an emergency communication system is the most basic and important means to win initiative, reduce losses and save lives and property. It is urgent to establish a modern and reliable emergency communication system. Emergency communication system not only requires strong mobility, but also needs the supporting measures of rapid and fast networking. In order to reduce the loss of people's lives and property, people realize the importance and urgency of improving the emergency communication system. Emergency communication refers to the mechanism of realizing communication by synthetically utilizing all kinds of communication resources and ensuring the means and methods of communication for rescuing, emergency aids and necessary communication when there is a natural or man-made sudden emergency situation. Emergency communication is not an independent new technology, but a comprehensive use of various communication technologies and means in emergency situations, the core of which is communication in emergency incidents. Emergency communication system is an important part of emergency communication system, which is produced to meet the communication needs in all kinds of emergency situations.

\section{Characteristics of emergency communication system}

Emergency communication system is produced to meet the communication needs of various emergency situations, but the location and scale of public security events such as natural disasters, especially social events, can not be predicted and prepared in advance. All kinds of emergencies have the following common features: The time required for emergency communication is generally uncertain, so people cannot prepare ahead of time for unexpected events such as earthquakes, tsunamis, fires, typhoons, mudslides, etc. The location of emergency communications is not sure; What type of network is required for emergency communications is undetermined. Emergency communications in different emergency situations have the following main attributes:

(1) Uncertainty of time and place; (2) Uncertainty of capacity demand; (3) High timeliness of emergency communication; (4) Complexity of the environment; (5) The uncertain destruction degree of the communication network; (6) Diversification of information (voice, SMS, image, video, etc.). 
Since emergency communication systems are different from daily communications systems and are mainly used for emergency communications in disasters and major emergencies, emergency communications systems should have the following characteristics:

(1) Miniaturization; (2) Quick layout and easy installation; (3) Energy-saving; (4)Mobility; (5) Simple and easy to operate; (6) Flexible structure; (7) Good system robustness; (8) System confidentiality; (9)Support multiple services.

\section{Research on emergency communication system}

\subsection{Satellite communication systems}

The construction of satellite communication system is utilized for emergency communication voice, data and image transmission under special circumstances. In the case that the ground network cannot be covered or the base station of the wireless communication network is destroyed, can't be replaced by the terrestrial communication network, which can provide broadband multimedia communication services, transmit video service, hold video conference, carry out emergency command such as quick rescue and processing in time, fast and reliably in any case. Due to the special needs of emergency communications, most of the characteristics of satellite communications can be the advantages of applied to emergency communications:

A wide range of communications. In case of emergency, the satellite communication terminal can communicate with the terminal of any place in the coverage area of the satellite beam or the command and dispatch center to transmit information such as sound, data, video and so on.

Since satellites are in space, they remain unaffected when natural disasters occur and when ground communications facilities are damaged.

The speed of construction is fast, and it is not easy to be restricted by geographical conditions. In the event of a disaster, satellite communication terminals can be rapidly deployed.

\subsection{Shortwave communications}

As a wireless communication mode, shortwave communication is equipped with strong antidestruction ability, long communication distance, rapid response and so on. When emergency scene and safety area need communication, as long as there are battery or small generator, the short wave radio can be deployed, transmitted and received, and can realize the short wave communication in the movement through the vehicle short wave equipment.

\subsection{Digital trunking communications}

Digital trunking communication system is an independent private network, not relying on the public communication network. It is an advanced wireless dispatching and command system with multi-use, high efficiency and low cost, which can share resources, share expenses and provide excellent service to users. The method of using digital trunking mobile phone is similar to that of conventional interphone, while conventional interphone communicates in fixed frequency, and digital trunking communication is multi-user sharing multi-frequency. In the emergency communication coverage area, the command and rescue personnel completing voice communication, voice dispatch and mobile transmission of data can adopt the digital trunking communication to provide an important means of communication for dealing with emergency situations. Digital trunking communication greatly saves the frequency resources, and its coverage and function is much larger than the conventional interphone.

The traditional emergency communication is mainly to protect emergency relief and communication network failure emergency. Although it has played a certain role in major events over the years, it also highlights its shortcomings. According to their own needs, the team built a network with the prominent geographical separation and, interconnection, and digital trunking communication technology is used in emergency communications, in addition to specificity, immediacy, mobility and so on, data transmission is also its strong strengh.

\subsection{Use of new communication technologies for communications}

When the terrain is more complex, the lines are difficult to set up, or the areas are not easy to communicate, such as mountain area, stratospheric communication can be taken advantage to meet the requirements of the emergency command communication. 
When it is necessary to provide the point to point integrated service wireless access of packet data service and circuit service, and support packet data, voice and video services based on IP, WiMAX can be adopted. Mobile WiMAX technology, with its advantages of fast networking, high speed and large capacity, provides a fast and reliable broadband visual emergency communication system.

\section{Summary}

The environment of emergency scene is complex and changeable, which is unpredictable. In order to ensure the effective communication of emergency scene, the realization of a highly efficient and reliable integrated emergency communication system adapted to various emergency sites is an important guarantee to improve the capacity of emergency field communication support. Under the condition of information, only by combining the changing situation at home and abroad, strengthening the further research and exploration of emergency communication, can we make the emergency communication more perfect and practical.

\section{References}

[1] Min Huang. Yaxin Wang. System Components and Applications of Emergency. Communication Vehicle. Telecommunications Technology, 2009.12.

[2]Peng Zhang,Jinying Chen. Unexpected Natural Disasters and Emergency Communications [J] Chengdu: School of information engineering, Chengdu University of Technology, 2009-1.

[3]Yiming Wang. Application of Satellite Communication in Emergency. Satellite and Network [J]2010-05.

[4] Xueli Zhang, Rui Wang. New Technology and System Application of Emergency Communication [M]Machinery Industry Press, 2010-1 